\title{
One-atom correlated-emission laser
}

\author{
Ho-Joon Kim, ${ }^{1,2}$ Ashfaq H. Khosa, ${ }^{1}$ Hai-Woong Lee, ${ }^{1,2}$ and M. Suhail Zubairy ${ }^{1,3}$ \\ ${ }^{1}$ Centre for Quantum Physics, COMSATS Institute of Information Technology, Islamabad, Pakistan \\ ${ }^{2}$ Department of Physics, Korea Advanced Institute of Science and Technology, Daejeon 305-701, Korea \\ ${ }^{3}$ Institute for Quantum Studies and Department of Physics, Texas A \& M University, College Station, Texas 77843-4242, USA \\ and Texas A \& M University at Qatar, Education City, P.O. Box 23874, Doha, Qatar
}

(Received 31 October 2007; published 14 February 2008)

\begin{abstract}
We study a four-level double- $\Lambda$ atomic configuration working as a two photon linear amplifier where two atomic transitions independently interact with cavity mode, while the other transitions are driven by a strong pump field. It is found that our system always works as a phase sensitive linear amplifier with no window for a phase insensitive linear amplifier. We also investigate that the system behaves as a two-photon correlatedemission laser under certain conditions.
\end{abstract}

DOI: 10.1103/PhysRevA.77.023817

PACS number(s): 42.50.Lc, 32.80.-t, 42.50.Ct

\section{INTRODUCTION}

A one-atom laser, a device that utilizes a trapped atom for the amplification of the laser field, is a system of interest in quantum optics. In the last decade there have been many theoretical proposals [1-5] as well as experimental demonstration [6-8] for the realizing of such a device. These experiments have utilized a beam of atoms in such a way that only one atom at a given time is responsible for lasing [6]. Contrary to this, McKeever et al. [9] were the first to have demonstrated the lasing of a single trapped atom. The same group also presented the theoretical analysis of their experiment [10].

The one-atom laser operation needs small mode volume $[1,3]$ which demands high cavity finesse so that the "strong coupling regime" conditions may be obtained. In this regime, Rabi frequency of the atom is much greater than both the atomic spontaneous emission rate and the cavity decay rate causing the atom to undergo several Rabi cycles before the decays. Theoretical predictions, from a full quantum description of the dynamics, are significantly different from the semiclassical laser models [3]. The investigation of one atom working as a source of a laser field is of fundamental interest as it allows one to test certain predictions of quantum optics. For example, in Ref. [11], Pellizzari and Ritsch showed that the stationary state of a one-atom Raman laser can get very close to a photon number eigenstate. Although the output of a one-atom laser is not a coherent state, it consists of a nonclassical state of radiation $[1,12]$. In a single atom laser one can observe photon antibunching as well as sub-Poissonian photon statistics [1] with coincidence measurements, particularly at small pump rates [4]. The emission is weak as compared with the laser standards and can be helpful in future quantum information schemes [9].

Here we study the atomic coherence effects in a one-atom laser which are the basis of many interesting phenomenon ranging from the correlated emission laser [13] to quantum informatics. In the subject of correlated emission laser the phase sensitive optical amplifiers [13,14] exhibit a wide range of possibilities regarding the amplification of a signal and the suppression of noise to continuous variable entanglement [14]. We know that one important source of noise in lasers is spontaneously emitted photons. In this case if the two spontaneous emission events are correlated then it is possible to quench the noise under certain conditions. In this way the linewidth of the laser can be suppressed bellow the Schawlow-Townes limit $[15,16]$. There are many systems proposed for the correlated emission laser $[13,17,18]$. In these systems usually the Fokker-Planck equation in $P$-representation [19] is used to find the quantum noise quenching condition, i.e., the vanishing of the diffusion coefficient for the relative phase at its phase locking point under certain conditions $[17,20]$. We also adopt the same methodology for finding the noise quenching parameters in our proposal.

It has been found recently that the correlated emission laser has the important property of producing a continuous source of the two-mode entanglement $[14,21,22]$ at macro scale. It has been shown that a two-mode correlated emission laser can lead to two-mode entanglement even when the average photon number in the field modes is very large $[21,22]$. In fact Kiffner $e t$ al. studied the atomic system closer to our system and showed the entanglement between the two different cavity fields [14].

In this paper we present a study of the four-level double$\Lambda$ atomic system serving as a two-photon linear amplifier and a correlated-emission laser. In our system two atomic transitions are driven by strong classical fields and the other two transitions are resonant to the cavity mode (i.e., we consider the case when the two atomic transitions interacting with the cavity are of the same frequency). The configuration we consider is, however, a "closed loop four-level atomic configuration." The same atomic configuration is discussed in many papers with different perspectives $[9,10,14]$. In this paper we find the parameters where the noise quenching can be achieved and present a detailed analysis of the equations obtained in our case. In this analysis both the resonant and off-resonant case of atom-field interaction are discussed.

\section{MASTER EQUATION FOR THE FIELD DENSITY OPERATOR}

Our system consists of a single four-level atom trapped in a cavity. The atom interacts with two classical fields acquir- 


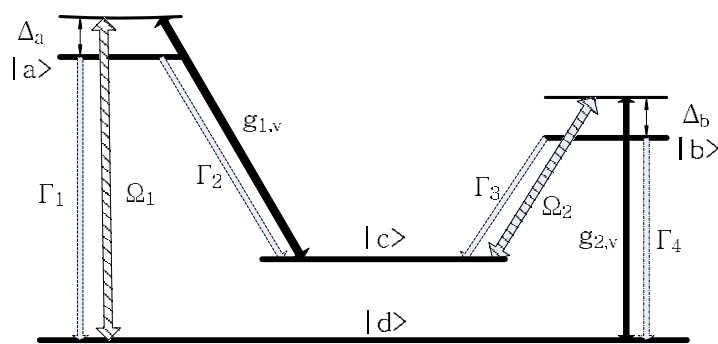

FIG. 1. (Color online) Two-photon linear amplifier atomic configuration showing its coupling with the pumping lasers and the cavity modes of the cavity.

ing energy and interacts with the cavity field to amplify it. Atomic level structure is shown in Fig. 1 where the transitions $|a\rangle \leftrightarrow|c\rangle$ and $|b\rangle \leftrightarrow|d\rangle$, with transition frequencies labeled as $w_{a c}$ and $w_{b d}$, interact with the cavity mode having coupling constant $g_{1}$ and $g_{2}$. These two transitions have detuning $\Delta_{2}=v_{2}-w_{a c}$ and $\Delta_{4}=v_{4}-w_{b d}$, respectively, where $v_{2}$ and $v_{4}$ are the cavity resonance frequencies. The atomic transitions $|a\rangle \leftrightarrow|d\rangle$ and $|b\rangle \leftrightarrow|c\rangle$ are labeled as $w_{a d}$ and $w_{b c}$ and are driven by a strong classical field of frequency $\omega_{1}$ and $\omega_{3}$, respectively. The field $\omega_{1}$ and the atomic transition $w_{a d}$ have detuning $\Delta_{1}=\omega_{1}-w_{a d}$. Similarly the field $\omega_{3}$ and atomic transition frequency $w_{b c}$ are mismatched by detuning $\Delta_{3}$ $=\omega_{3}-w_{b c}$.

It is clear from Fig. 1 that our system is a closed loop system as stated earlier. For the study of such a system, we first derive the equation of motion for the reduced density operator of the cavity field where the free part of the Hamiltonian for the field and the atom are $H_{F}=\hbar v a^{\dagger} a$ and $H_{A}$ $=\sum_{i=a, b, c, d} \hbar \omega_{i}|i\rangle\langle i|$, respectively. For simplicity sake, we have considered the situation where the atomic transitions from $|a\rangle \leftrightarrow|c\rangle$ and $|b\rangle \leftrightarrow|d\rangle$ have equal frequencies (i.e., $v_{2}$ $=v_{4}=v$ ).

The interaction part of the Hamiltonian consists of two parts. One is related to the $|a\rangle \leftrightarrow|c\rangle$ and $|b\rangle \leftrightarrow|d\rangle$ transitions and is the so-called cavity Hamiltonian. Under dipole and rotating wave approximation we can write this part of the Hamiltonian as

$$
H_{C}=\hbar a\left(g_{1}|a\rangle\left\langle c\left|+g_{2}\right| b\right\rangle\langle d|\right)+\text { H.c. },
$$

where $g_{1}$ and $g_{2}$ are the vacuum Rabi frequencies and $a\left(a^{\dagger}\right)$ is the annihilation (creation) operator of the field mode. It is to be noted that in the following calculations we take $g_{1}$ and $g_{2}$ as real quantities. The second part of the Hamiltonian governing the interaction of the atom and the classical fields (denoted by $H_{L}$ ) is given by

$$
H_{L}=-\hbar \Omega_{1} e^{-i \omega_{1} t}|a\rangle\left\langle d\left|-\hbar \Omega_{2} e^{-i \omega_{3} t}\right| b\right\rangle\langle c|+\text { H.c. },
$$

where $\Omega_{1}$ and $\Omega_{2}$ are the Rabi frequencies of the driving fields. In general the Rabi frequencies are complex, i.e., $\Omega_{1}$ $=\left|\Omega_{1}\right| e^{-i \phi_{1}}, \Omega_{2}=\left|\Omega_{2}\right| e^{-i \phi_{2}}$. The equation of motion for the atom-field density operator is given by

$$
\frac{d \rho}{d t}=-\frac{1}{i \hbar}\left[\rho, H_{A}+H_{F}+H_{C}+H_{L}\right]+L_{\gamma}(\rho),
$$

where the last term represents decay and reads

$$
L_{\gamma}(\rho)=-\frac{1}{2} \sum_{i=1}^{4} \Gamma_{i}\left(\sigma_{i}^{\dagger} \sigma_{i} \rho+\rho \sigma_{i}^{\dagger} \sigma_{i}-2 \sigma_{i} \rho \sigma_{i}^{\dagger}\right) .
$$

The atomic transition operators $\sigma_{1}=|d\rangle\left\langle a\left|, \sigma_{2}=\right| c\right\rangle\langle a|, \sigma_{3}$ $=|c\rangle\langle b|$, and $\sigma_{4}=|d\rangle\langle b|$ denote the corresponding transitions in the atomic internal state and $\Gamma_{i}$ are the decay rates of these atomic transitions.

\section{Reduced density operator}

In this section we obtain the equation of motion for the reduced density operator of the cavity field. For convenience, we transform the Schrödinger picture density operator into the interaction picture via $\hat{W}=\hat{W}_{F} \otimes \hat{W}_{A}$ where $\hat{W}_{F}$ and $\hat{W}_{A}$ are the unitary operators corresponding to the field and the atom, respectively. In the expression of unitary operator corresponding to the atom we also add detunings of the atomic levels. Under this transformation the density operator is given as $\widetilde{\rho}=\hat{W} \rho \hat{W}^{\dagger}$, where we put a tilde to mark the new frame. Since our configuration consists of a closed loop, we consider $\Delta_{1}=\Delta_{2}=\Delta_{a}$, and $\Delta_{3}=\Delta_{4}=\Delta_{b}$, which insures that the two photon resonance condition is fulfilled. This also ensures that the Hamiltonian will be time independent in the interaction picture.

From the governing equation of motion for the density matrix

$$
\frac{d \widetilde{\rho}}{d t}=-\frac{1}{i \hbar}\left[\tilde{\rho}, H_{0}+H_{C}\right]+L_{\gamma}(\widetilde{\rho}),
$$

we obtain the equation of motion of the reduced density matrix for the cavity field using $\frac{d}{d t}\left(\widetilde{\rho}_{F}\right)=\operatorname{Tr}_{A}\left(\frac{d \widetilde{\rho}}{d t}\right)$, which yields

$$
\frac{d \widetilde{\rho}_{F}}{d t}=-i g_{1}\left[a^{\dagger}, \widetilde{\rho}_{a c}\right]-i g_{2}\left[a^{\dagger}, \widetilde{\rho}_{b d}\right]+\text { H.c. }
$$

It is clear from Eq. (6) that for the reduced density matrix $\tilde{\rho}_{F}$, we need $\tilde{\rho}_{a c}, \tilde{\rho}_{b d}$, and their Hermitian conjugates. To get these expressions we return to Eq. (5) and solve it for $\tilde{\rho}_{a c}$, $\tilde{\rho}_{b d}$, and their Hermitian conjugates. During solving for these parameters we retain only those terms that are first order in coupling constants $g_{1}$ and $g_{2}$. Also we expand $\tilde{\rho}$ in Eq. (5) up to first order, $\widetilde{\rho}=\widetilde{\rho}_{0}+\widetilde{\rho}_{C}$, and consider that $H_{C}$ is first order in $g_{1}$ and $g_{2}$. The resulting uncoupled differential equations are

$$
\frac{d \widetilde{\rho}_{0}}{d t}=L_{0}\left(\widetilde{\rho}_{0}\right),
$$

$$
\frac{d \widetilde{\rho}_{C}}{d t}=-\frac{1}{i \hbar}\left[\widetilde{\rho}_{0}, H_{C}\right]+L_{0}\left(\widetilde{\rho}_{C}\right),
$$

where 


$$
L_{0}(\cdot)=-\frac{1}{i \hbar}\left[\cdot, H_{0}\right]+L_{\gamma}(\cdot) .
$$

Equation (7) is the zeroth-order equation which describes the interactions of the atom with the classical laser fields and Eq. (8) is the first-order equation governing the cavity mode and the damping terms. We find the steady-state $\left(\dot{\tilde{\rho}}_{0}=0\right)$ solution of Eq. (7) for $\tilde{\rho}_{0}$ with the condition $\operatorname{Tr}_{A}\left(\tilde{\rho}_{0}\right)=\tilde{\rho}_{F}$. Inserting this solution into Eq. (8) we obtain the steady-state solution of Eq. (8) for $\tilde{\rho}_{C}$. With these two solutions $\left(\tilde{\rho}_{0}\right.$ and $\left.\tilde{\rho}_{C}\right)$ we find

$$
\begin{aligned}
& i g_{1} \tilde{\rho}_{a c}=\alpha_{11} a \widetilde{\rho}_{F}+\alpha_{12} a^{\dagger} \widetilde{\rho}_{F}+\beta_{11} \widetilde{\rho}_{F} a+\beta_{12} \widetilde{\rho}_{F} a^{\dagger}, \\
& i g_{2} \widetilde{\rho}_{b d}=\alpha_{22} a \widetilde{\rho}_{F}+\alpha_{21} a^{\dagger} \widetilde{\rho}_{F}+\beta_{22} \widetilde{\rho}_{F} a+\beta_{21} \tilde{\rho}_{F} a^{\dagger} .
\end{aligned}
$$

Here the coefficients $\alpha_{i j}$ and $\beta_{i j}$ are given in the Appendix. It follows on substituting Eq. (10) into Eq. (6) that

$$
\begin{aligned}
\frac{d}{d t} \widetilde{\rho}_{F}= & \left(\beta_{11}^{*}+\beta_{22}^{*}\right) a a^{\dagger} \widetilde{\rho}_{F}+\left(\beta_{11}+\beta_{22}\right) \widetilde{\rho}_{F} a a^{\dagger}-\left(\alpha_{11}+\alpha_{22}\right) a^{\dagger} a \widetilde{\rho}_{F}-\left(\alpha_{11}^{*}+\alpha_{22}^{*}\right) \widetilde{\rho}_{F} a^{\dagger} a+\left(\beta_{12}^{*}+\beta_{21}^{*}\right) a a \widetilde{\rho}_{F}+\left(\beta_{12}+\beta_{21}\right) \widetilde{\rho}_{F} a^{\dagger} a^{\dagger} \\
& -\left(\alpha_{12}^{*}+\alpha_{21}^{*}\right) \widetilde{\rho}_{F} a a-\left(\alpha_{12}+\alpha_{21}\right) a^{\dagger} a^{\dagger} \widetilde{\rho}_{F}-\left(\beta_{11}^{*}+\beta_{22}^{*}+\beta_{11}+\beta_{22}\right) a^{\dagger} \widetilde{\rho}_{F} a+\left(\alpha_{11}+\alpha_{22}+\alpha_{11}^{*}+\alpha_{22}^{*}\right) a \widetilde{\rho}_{F} a^{\dagger} \\
& -\left(\beta_{12}^{*}+\beta_{21}^{*}-\alpha_{12}^{*}-\alpha_{21}^{*}\right) a \widetilde{\rho}_{F} a+\left(\alpha_{12}+\alpha_{21}-\beta_{12}-\beta_{21}\right) a^{\dagger} \widetilde{\rho}_{F} a^{\dagger} .
\end{aligned}
$$

Equation (11) provides the bases for further calculation where we study the lasing properties of our system.

\section{TWO-PHOTON LINEAR AMPLIFIER}

This section evaluates the noise and gain terms for two conjugate quadratures so that we may examine our system as a two-photon linear amplifier. We make a system of firstorder differential equations for expectation values of the operators $a, a^{\dagger}, a^{\dagger} a$, and $a a$, which are required to calculate the variance of quadratures. Starting with the equation of motion for the expectation values (i.e., $\quad \frac{d}{d t}\langle O\rangle=\frac{d}{d t} \operatorname{Tr}\left[O \tilde{\rho}_{F}\right]$ $=\operatorname{Tr}\left[O \frac{d}{d t} \tilde{\rho}_{F}\right]$ ), Eq. (11) provides the differential equations for the required operators

$$
\begin{gathered}
\frac{d\langle a\rangle}{d t}=\rho_{1}\langle a\rangle+\rho_{2}\left\langle a^{\dagger}\right\rangle, \\
\frac{d\left\langle a^{\dagger} a\right\rangle}{d t}=\left(\rho_{1}+\rho_{1}^{*}\right)\left\langle a^{\dagger} a\right\rangle+\rho_{2}^{*}\langle a a\rangle+\rho_{2}\left\langle a^{\dagger} a^{\dagger}\right\rangle+\rho_{11}, \\
\frac{d\langle a a\rangle}{d t}=2 \rho_{1}\langle a a\rangle+2 \rho_{2}\left\langle a^{\dagger} a\right\rangle+2 \rho_{12},
\end{gathered}
$$

where

$$
\begin{gathered}
\rho_{1}=-\left(\alpha_{11}+\alpha_{22}+\beta_{11}+\beta_{22}\right), \\
\rho_{2}=-\left(\alpha_{12}+\alpha_{21}+\beta_{12}+\beta_{21}\right), \\
\rho_{11}=-\left(\beta_{11}+\beta_{22}+\beta_{11}^{*}+\beta_{22}^{*}\right), \\
\rho_{12}=-\left(\alpha_{12}+\alpha_{21}\right) .
\end{gathered}
$$

This set of equations of motion for the expectation values [Eqs. (12)-(14)] have the same structure as was derived by
Ansari, Gea-Banacloche, and Zubairy in Ref. [13]. However, in our case the coefficients of these operators have a different structure. It is well-known that the system of equations from Eqs. (12) and (14) are exactly solvable. Here we discuss two special cases of resonant and off-resonant atom-field interactions.

\section{Resonant case}

For the sake of computational simplicity we take decay rates of different atomic level to be equal, i.e., $\Gamma_{1}=\Gamma_{2}=\Gamma_{3}$ $=\Gamma_{4}=\Gamma$. In this case $\rho_{1}$ becomes equal to zero for $\Delta_{a}=\Delta_{b}$ $=0$. We find the expressions for $\rho_{2}, \rho_{11}$, and $\rho_{12}$ under these conditions, i.e.,

$$
\begin{gathered}
\rho_{2}=\frac{2 \Gamma g_{1} g_{2} \Omega_{1} \Omega_{2}}{P_{6}}, \\
\rho_{11}=\frac{2 \Gamma\left(g_{1}^{2}+g_{2}^{2}\right)\left|\Omega_{1}\right|^{2}\left|\Omega_{2}\right|^{2}\left(2 \Gamma^{2}+\left|\Omega_{1}\right|^{2}+\left|\Omega_{2}\right|^{2}\right)}{P_{6} P_{7}}, \\
\rho_{12}=\frac{2 \Gamma g_{1} g_{2} \Omega_{1} \Omega_{2}\left(P_{7}-4\left|\Omega_{1}\right|^{2}\left|\Omega_{2}\right|^{2}\right)}{P_{6} P_{7}},
\end{gathered}
$$

where $P_{6}=\Gamma^{2}\left(\left|\Omega_{1}\right|^{2}+\left|\Omega_{2}\right|^{2}\right)+4\left|\Omega_{1}\right|^{2}\left|\Omega_{2}\right|^{2}$ and $P_{7}=\left(\left|\Omega_{1}\right|^{2}\right.$ $\left.-\left|\Omega_{2}\right|^{2}\right)^{2}+2 \Gamma^{2}\left(\left|\Omega_{1}\right|^{2}+\left|\Omega_{2}\right|^{2}\right)$. Now we assume that $\rho_{2}$ is a real number, i.e., $\rho_{2}=\rho_{2}$. In this case Eq. (19) shows that the product of Rabi frequencies $\Omega_{1} \Omega_{2}=\left|\Omega_{1}\right| e^{-i \phi_{1}}\left|\Omega_{2}\right| e^{-i \phi_{2}}$ $\rightarrow\left|\Omega_{1}\right|\left|\Omega_{2}\right| e^{-i\left(\phi_{1}+\phi_{2}\right)}$. This suggests that by adjusting the phases of the classical fields we can control phase sensitive amplification. This is possible when $\phi_{1}=-\phi_{2}$ so that the imaginary part of the equation always vanishes. By taking $\rho_{2}$ a real number also makes $\rho_{12}$ real. In this case, Eqs. (12)-(14) attain the simple form

$$
\frac{d\langle a\rangle}{d t}=\rho_{2}\left\langle a^{\dagger}\right\rangle
$$




$$
\begin{gathered}
\frac{d\left\langle a^{\dagger} a\right\rangle}{d t}=\rho_{2}\langle a a\rangle+\rho_{2}\left\langle a^{\dagger} a^{\dagger}\right\rangle+\rho_{11}, \\
\frac{d\langle a a\rangle}{d t}=2 \rho_{2}\left\langle a^{\dagger} a\right\rangle+2 \rho_{12} .
\end{gathered}
$$

The resulting solutions are

$$
\begin{gathered}
\langle a\rangle_{t}=\langle a\rangle_{0} \cosh \left(\rho_{2} t\right)+\left\langle a^{\dagger}\right\rangle_{0} \sinh \left(\rho_{2} t\right) \\
\left\langle a^{\dagger} a\right\rangle_{t}=\left\langle a^{\dagger} a\right\rangle_{0} \cosh \left(2 \rho_{2} t\right)+\frac{\langle a a\rangle_{0}}{2} \sinh \left(2 \rho_{2} t\right) \\
+\frac{\left\langle a^{\dagger} a^{\dagger}\right\rangle_{0}}{2} \sinh \left(2 \rho_{2} t\right)+\frac{\rho_{11}}{2 \rho_{2}} \sinh \left(2 \rho_{2} t\right) \\
+\frac{\rho_{12}}{\rho_{2}}\left\{\cosh \left(2 \rho_{2} t\right)-1\right\} \\
\langle a a\rangle_{t}=\left\langle a^{\dagger} a\right\rangle_{0} \sinh \left(2 \rho_{2} t\right)+\frac{\langle a a\rangle_{0}}{2}\left\{\cosh \left(2 \rho_{2} t\right)+1\right\} \\
+\frac{\left\langle a^{\dagger} a^{\dagger}\right\rangle_{0}}{2}\left\{\cosh \left(2 \rho_{2} t\right)-1\right\}+\frac{\rho_{11}}{2 \rho_{2}}\left\{\cosh \left(2 \rho_{2} t\right)-1\right\} \\
+\frac{\rho_{12}}{\rho_{2}} \sinh \left(2 \rho_{2} t\right)
\end{gathered}
$$

With the above solution in hand, we consider the variances of two quadratures defined by $X_{1}=\frac{1}{2}\left(a+a^{\dagger}\right)$ and $X_{2}$ $=\frac{1}{2 i}\left(a-a^{\dagger}\right)$. The expectation values for these quadratures are given by

$$
\begin{aligned}
& \left\langle X_{1}\right\rangle_{t}=\sqrt{G_{1}(t)}\left\langle X_{1}\right\rangle_{0}, \\
& \left\langle X_{2}\right\rangle_{t}=\sqrt{G_{2}(t)}\left\langle X_{2}\right\rangle_{0},
\end{aligned}
$$

where

$$
G_{1}(t)=\exp \left(2 \rho_{2} t\right), \quad G_{2}(t)=\exp \left(-2 \rho_{2} t\right)
$$

are identified as the gain terms in defined quadratures, respectively. Our system is therefore phase-sensitive for nonvanishing $\rho_{2}$. The variances of two quadratures are obtained using the standard methods discussed in Ref. [16],

$$
\begin{aligned}
& \left(\Delta X_{1}\right)_{t}^{2}=G_{1}\left(\Delta X_{1}\right)_{0}^{2}+N_{1}\left(G_{1}-1\right), \\
& \left(\Delta X_{2}\right)_{t}^{2}=G_{2}\left(\Delta X_{2}\right)_{0}^{2}+N_{2}\left(G_{2}-1\right),
\end{aligned}
$$

where

$$
\begin{aligned}
& N_{1}=\frac{2 \rho_{12}-\rho_{2}+\rho_{11}}{4 \rho_{2}}, \\
& N_{2}=\frac{2 \rho_{12}-\rho_{2}-\rho_{11}}{4 \rho_{2}}
\end{aligned}
$$

show the noise dependence in the two conjugate quadratures. When $\rho_{2}>0$, we have $N_{1}>N_{2}$. Similarly, when $\rho_{2}<0$, we have $N_{1}<N_{2}$ and this is only possible when $e^{-i\left(\phi_{1}+\phi_{2}\right)}=-1$.

Regarding $g \propto \sqrt{\nu}$ and the fact that $g_{1}$ and $g_{2}$ occur in a symmetric way in the expression of $G_{1}, G_{2}, N_{1}$, and $N_{2}$, we make one more assumption, namely, $g_{1}=g_{2}=g$ which does not effect our analysis. The resulting simple expressions for gains and noises are

$$
\begin{gathered}
G_{1}(t)=\exp \left\{\frac{4 t \Gamma\left|\Omega_{1}\right|\left|\Omega_{2}\right| g^{2}}{P_{6}}\right\}, \\
G_{2}(t)=\exp \left\{-\frac{4 t \Gamma\left|\Omega_{1}\right|\left|\Omega_{2}\right| g^{2}}{P_{6}}\right\}, \\
N_{1}=\frac{1}{4}+\frac{\left|\Omega_{1}\right|\left|\Omega_{2}\right|\left\{2 \Gamma^{2}-2\left|\Omega_{1}\right|\left|\Omega_{2}\right|+\left(\left|\Omega_{1}\right|-\left|\Omega_{2}\right|\right)^{2}\right\}}{2 P_{7}}, \\
N_{2}=\frac{1}{4}-\frac{\left|\Omega_{1}\right|\left|\Omega_{2}\right|\left\{2 \Gamma^{2}+2\left|\Omega_{1}\right|\left|\Omega_{2}\right|+\left(\left|\Omega_{1}\right|+\left|\Omega_{2}\right|\right)^{2}\right\}}{2 P_{7}} .
\end{gathered}
$$

Next we investigate the gain and noise for a few typical regions.

\section{When any one of the Rabi frequencies is zero}

In the absence of both driving fields our system becomes two two-level systems interacting with the same cavity field where the upper state decays to the lower state, respectively. Since there is no source of external excitation we do not have any gain after the whole system reaches its steady state. The equation of motion for the cavity field is $d \rho_{F} / d t=0$. In case any of the Rabi frequencies is equal to zero, we see from Eq. (19) that $\rho_{2}=0$. Therefore from Eqs. (35)-(38), it is apparent that

$$
G_{1}=G_{2}=1, \quad N_{1}=N_{2}=\frac{1}{4},
$$

unless $\Gamma=0$. We have no gains and consequently no noise added because variances do not change.

\section{When Rabi frequencies are equal}

Here we consider a special case when both the Rabi frequencies are equal (i.e., $\left|\Omega_{1}\right|=\left|\Omega_{2}\right|=|\Omega|$ ). In this case the following results are obtained for the gain and noises:

$$
\begin{aligned}
G_{1} & =\exp \left(\frac{4 \Gamma g^{2}|\Omega|^{2} t}{2 \Gamma^{2}|\Omega|^{2}+4|\Omega|^{4}}\right) \\
& =\exp \left(\frac{2 \Gamma g^{2} t}{\Gamma^{2}+2|\Omega|^{2}}\right) \approx \exp \left(\frac{\Gamma g^{2} t}{|\Omega|^{2}}\right), \quad|\Omega| \gg \Gamma, \\
& \approx \exp \left(\frac{2 g^{2} t}{\Gamma}\right), \quad|\Omega| \ll \Gamma,
\end{aligned}
$$




$$
\begin{aligned}
& G_{2}=\exp \left(-\frac{4 \Gamma g^{2}|\Omega|^{2} t}{2 \Gamma^{2}|\Omega|^{2}+4|\Omega|^{4}}\right) \\
& =\exp \left(-\frac{2 \Gamma g^{2} t}{\Gamma^{2}+2|\Omega|^{2}}\right) \approx \exp \left(-\frac{\Gamma g^{2} t}{|\Omega|^{2}}\right), \quad|\Omega| \gg \Gamma, \\
& \approx \exp \left(-\frac{2 g^{2} t}{\Gamma}\right), \quad|\Omega| \ll \Gamma \text {, } \\
& N_{1}=\frac{1}{4}+\frac{|\Omega|^{2}\left(2 \Gamma^{2}-2|\Omega|^{2}\right)}{8 \Gamma^{2}|\Omega|^{2}}=\frac{1}{4}+\frac{\Gamma^{2}-|\Omega|^{2}}{4 \Gamma^{2}} \\
& \approx-\frac{|\Omega|^{2}}{4 \Gamma^{2}}, \quad|\Omega| \gg \Gamma, \\
& N_{1} \rightarrow-\infty \quad \text { when } \Gamma \rightarrow 0 \\
& \approx \frac{1}{2}, \quad|\Omega| \ll \Gamma, \\
& N_{2}=\frac{1}{4}-\frac{|\Omega|^{2}\left(2 \Gamma^{2}+6|\Omega|^{2}\right)}{8 \Gamma^{2}|\Omega|^{2}} \\
& =\frac{1}{4}-\frac{\Gamma^{2}+3|\Omega|^{2}}{4 \Gamma^{2}} \\
& \approx-\frac{3|\Omega|^{2}}{4 \Gamma^{2}} \rightarrow-\infty, \quad|\Omega| \gg \Gamma, \\
& N_{2} \rightarrow-\infty \quad \text { when } \Gamma \rightarrow 0 \text {, } \\
& \approx 0, \quad|\Omega| \ll \Gamma .
\end{aligned}
$$

We see (for $\rho_{2}>0$ ) that $G_{1}>1, G_{2}<1$, and $G_{1}=1 / G_{2}$ always hold. If $|\Omega| \ll \Gamma$, it becomes a peculiar phasesensitive linear amplifier; $X_{1}$ amplified whereas $X_{2}$ deamplified, with noise only in the $X_{1}$ quadrature. If $|\Omega| \gg \Gamma, X_{1}$ is amplified whereas $X_{2}$ deamplified, but unequal infinite minus noises accompanied into both the quadratures. In this case our system becomes a degenerate parametric amplifier [13]. The system behaves differently when $\rho_{2}<0$. The noise $N_{1}$ behaves like noise $N_{2}$ and Gain $G_{1}$ behaves like $G_{2}$, and vice versa, for a suitable choice of phase i.e., $\exp \left[-i\left(\phi_{1}+\phi_{2}\right)\right.$ $=1]$, see Figs. 2 and 3 .

\section{Other regions}

From the two special and extreme cases, one might guess the whole picture. For those cases we already have quite a few expressions there, though it is apparent that we will encounter more formidable expressions in other regions. In that sense, a glimpse to the graphs gives us an insight. Here we present graphs of gains and noises under the conditions used hitherto; $\Delta_{a}=\Delta_{b}=0, \Gamma_{i}=\Gamma, g_{i}=g$, and $\Omega_{1} \Omega_{2}=\left|\Omega_{1}\right|\left|\Omega_{2}\right|$. For each $G_{i}, N_{i}$ we provide two graphs, one in the proximity of the origin and the other for a more broader view. This is because there are quite a few variations near the origin. Also note the ranges of each gain and noise. Parameters are determined regarding experimental conditions, for example, those in Ref. [9].
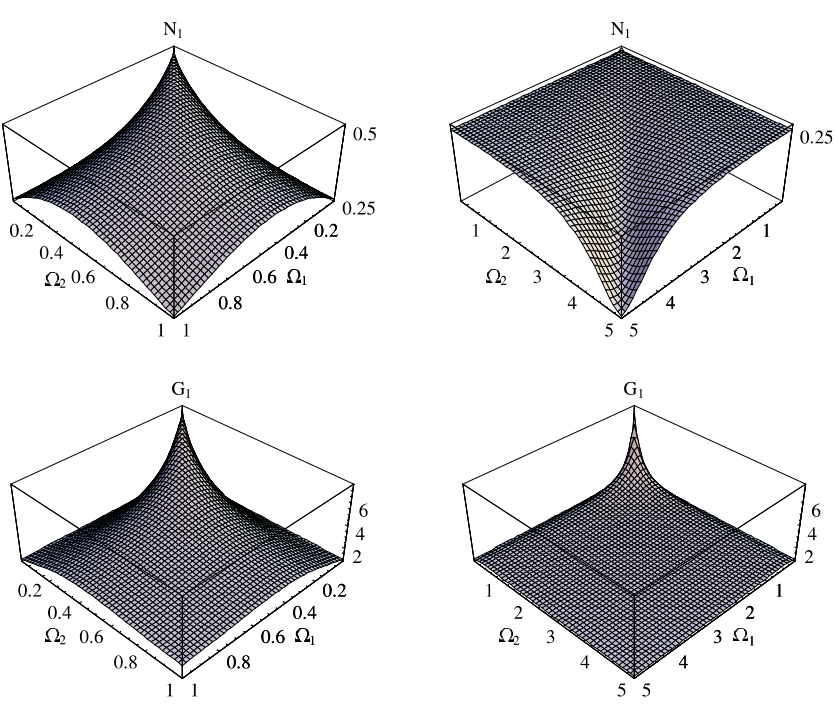

FIG. 2. (Color online) Gain and noise of quadrature $X_{1}$. We set $\Gamma=1 \mathrm{MHz}, g=10 \mathrm{MHz}$, and $t=10 \mathrm{~ns}$. For gain and noise there are two graphs, respectively: One in the proximity of the origin and the other for a more broader view.

In the previous work [17] (where atomic coherence in a three level cascaded model is initially injected), two quadratures obtained the same gain but different added noises. Also in Ref. [13] atomic coherence is induced by a driving field and the system behaves as a phase insensitive linear amplifier, i.e., equal gain and equal noise in both quadratures for $|\Omega| \ll \gamma$ whereas it becomes a parametric amplifier, i.e., different gains, $G_{1} G_{2}=1$, and vanishing noises in both quadratures for $|\Omega| \gg \gamma$. These results show that the way atomic coherence enters into a system leads to differences in the dynamics of the system. Compared to the above cited papers, our system is close to the second one since the atomic co-
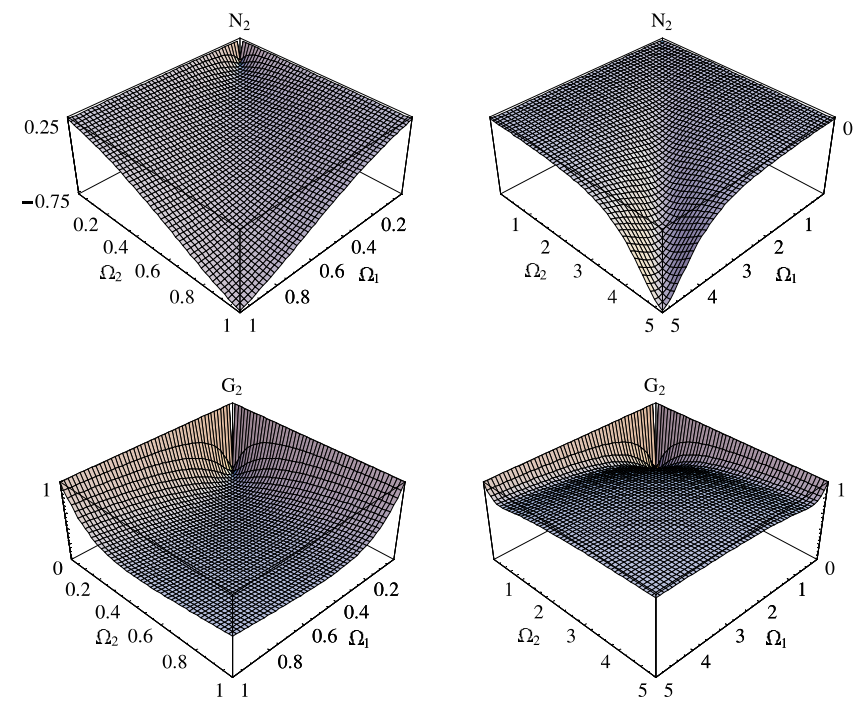

FIG. 3. (Color online) Gain and noise of quadrature $X_{2}$. We set $\Gamma=1 \mathrm{MHz}, g=10 \mathrm{MHz}$, and $t=10 \mathrm{~ns}$. For gain and noise there are two graphs, respectively: One in the proximity of the origin and the other for a more broader view. 
herence is induced by driving fields. However, as we see in the graphs, our system shows remarkably different behavior as compared with the other system. It always works as a phase sensitive linear amplifier with no window for a phase insensitive linear amplifier. However, it never obtains vanishing added noises for both quadratures as in the case for a parametric amplifier. Therefore it cannot reproduce the result of Ref. [13]. It also adds noise to amplified quadrature $X_{1}$ through preferred selection of phases of the driving fields, and no noise to deamplified quadrature $X_{2}$ so that it cannot reproduce the result of Ref. [17].

Another difference from the result of Ref. [13] occurs when there are no classical driving fields. The system in [13] behaves as a phase-insensitive amplifier while we do not have this result. The reason is that the injected atoms are prepared in an excited state which serves as a source of energy to the cavity field while our system does not have that kind of source in the absence of driving fields.

\section{Off-resonant case}

In this section, again we consider equal decay rates. For the case where $\Delta_{a} \neq \Delta_{b}, \rho_{1}$ is in general not zero, but if $\Delta_{a}$ $=\Delta_{b}$, then it becomes zero. Here, for the sake of simplicity, we consider an equal nonzero detuning case which gives the following results:

$$
\rho_{2}=\frac{2 g_{1} g_{2} \Omega_{1} \Omega_{2}(\Gamma+i \Delta)\left|\left(\Omega_{d}\right)^{2}+2 \Gamma^{2}\left(\Omega_{s}\right)-2 i \Delta \Gamma\left(\Omega_{d}\right)\right|^{2}}{\left(\Omega_{d}\right)^{2}\left\{4 \Gamma^{2}\left(\Omega_{s}+\Delta^{2}\right)+\left(\Omega_{d}\right)^{2}\right\}+4 \Gamma^{4}\left(\Omega_{s}\right)^{2}},
$$

$$
\begin{gathered}
\rho_{11}=\frac{2 \Gamma\left(g_{1}^{2}+g_{2}^{2}\right)\left|\Omega_{1}\right|^{2}\left|\Omega_{2}\right|^{2}\left[\Omega_{s}\left\{\left(\Omega_{d}\right)^{2}+4 \Gamma^{2}\left(\Gamma^{2}+\Delta^{2}+\Omega_{s}\right)\right\}-8 \Gamma^{2}\left|\Omega_{1}\right|^{2}\left|\Omega_{2}\right|^{2}\right]}{\left\{\left(\Gamma^{2}+\Delta^{2}\right) \Omega_{s}+4\left|\Omega_{1}\right|^{2}\left|\Omega_{2}\right|^{2}\right\}\left[\left(\Omega_{d}\right)^{2}\left\{\left(\Omega_{d}\right)^{2}+P_{9}\right\}-P_{8}\right]}, \\
\rho_{12}=\frac{2 g_{1} g_{2} \Omega_{1} \Omega_{2}\left\{\left(\Gamma^{2}+\Delta^{2}\right) \Omega_{s}+4\left|\Omega_{1}\right|^{2}\left|\Omega_{2}\right|^{2}\right\}\left[\left[\left(\Omega_{d}\right)^{2}\left\{\left(\Omega_{d}\right)^{2}+P_{9}\right\}-P_{8}\right]\right.}{\left\{\left(\Gamma^{2}+\Delta^{2}\right) \Omega_{s}+4\left|\Omega_{1}\right|^{2}\left|\Omega_{2}\right|^{2}\right\}\left[\left(\Omega_{d}\right)^{2}\left\{\left(\Omega_{d}\right)^{2}+P_{9}\right\}-P_{8}\right]},
\end{gathered}
$$

where $\quad \Omega_{d}=\left|\Omega_{1}\right|^{2}-\left|\Omega_{2}\right|^{2}, \quad \Omega_{s}=\left|\Omega_{1}\right|^{2}+\left|\Omega_{2}\right|^{2}, \quad P_{8}$ $=16 \Gamma^{2}\left|\Omega_{1}\right|^{2}\left|\Omega_{2}\right|^{2}\left(\Omega_{s}-\Gamma^{2}\right)$, and $P_{9}=4 \Gamma^{2}\left(\Omega_{d}+\Gamma^{2}+\Delta^{2}\right)$. Here the phase of $\rho_{2}$ is determined by $(\Gamma+i \Delta) \Omega_{1} \Omega_{2}$ so we can make $\rho_{2}$ real by adjusting the phases of driving fields as in the resonant case. This could be achieved by setting the imaginary part of $\rho_{2}$ [i.e., $\left.\Delta \cos \left(\phi_{1}+\phi_{2}\right)-\Gamma \sin \left(\phi_{1}+\phi_{2}\right)\right]$ in Eq. (46) equal to zero. According to this situation the sum of the phases can easily be adjusted so that the condition $\phi_{1}$ $+\phi_{2}=\tan ^{-1}(\Gamma / \Delta)$ should be fulfilled for any value of $\Gamma$ and $\Delta$. Therefore we take $\rho_{2}=\rho_{2}^{*}>0$ which is achievable by adjusting the phases of the driving fields. In this situation, for the off-resonant case with modified values of $\rho_{2}, \rho_{11}$, and $\rho_{12}$ [Eqs. (46)-(48)], we have exactly the same differential equations, as given in Eqs. (12)-(14) for the expectation values of different field operators. Hence we can straightforwardly get the expressions for the quadratures and relevant expressions for gains and variances. The noises in the conjugate quadratures in the off-resonant case are given by

$$
\begin{aligned}
& N_{1}=\frac{2 \operatorname{Re}\left[\rho_{12}\right]-\rho_{2}+\rho_{11}}{4 \rho_{2}}, \\
& N_{2}=\frac{2 \operatorname{Re}\left[\rho_{12}\right]-\rho_{2}-\rho_{11}}{4 \rho_{2}},
\end{aligned}
$$

showing explicitly the identical expressions as in the case of resonant atom-field interaction. Again we see that $g_{1}, g_{2}$ appear in a symmetric way so that we may assume $g_{1}=g_{2}=g$ without any problem. Basically we have similar results for gains and noises for a nonzero off-resonant case if we adjust driving fields appropriately. We show corresponding graphs for gains and noises.

\section{TWO PHOTON LASER}

In this section, we investigate the properties of our system as a correlated emission laser adding cavity decay terms which are essential for the purpose, i.e.,

$$
\left(\frac{d \rho}{d t}\right)_{\text {decay }}=-\gamma\left(a^{\dagger} a \rho_{F}+\rho_{F} a^{\dagger} a-2 a \rho_{F} a^{\dagger}\right)
$$

where $\gamma$ is the cavity decay rate. Since we do not consider the saturation of the gain medium our equations are valid in the linear regime below threshold. However, to a good approximation we can get information about phase drift and the diffusion term of steady state.

To convert our cavity decay term added master equation to a $c$-number Fokker-Planck equation in the $P$-representation, we use

$$
\begin{gathered}
a|\alpha\rangle\langle\alpha|=\alpha| \alpha\rangle\langle\alpha|, \\
a^{\dagger}|\alpha\rangle\left\langle\alpha\left|=\left(\frac{\partial}{\partial \alpha}+\alpha^{*}\right)\right| \alpha\right\rangle\langle\alpha| .
\end{gathered}
$$

Hence our system is described by 


$$
\begin{aligned}
\frac{\partial P}{\partial t}= & {\left[\left\{\left(\beta_{11}+\beta_{22}\right)\left(\frac{\partial}{\partial \alpha} \alpha-\frac{\partial^{2}}{\partial \alpha \partial \alpha^{*}}\right)+\left(\alpha_{11}+\alpha_{22}+\gamma\right) \frac{\partial}{\partial \alpha} \alpha\right.\right.} \\
& \left.+\left(\alpha_{12}+\alpha_{21}\right)\left(\frac{\partial}{\partial \alpha} \alpha^{*}-\frac{\partial^{2}}{\partial \alpha^{2}}\right)+\left(\beta_{12}+\beta_{21}\right) \frac{\partial}{\partial \alpha} \alpha^{*}\right\} \\
& + \text { c.c. }] P .
\end{aligned}
$$

We transform the above equation into the amplitude and phase variable $r$ and $\theta$ representation by considering $\alpha$ $=r e^{i \theta}$. The relevant operators can be written as

$$
\begin{gathered}
\frac{\partial}{\partial \alpha}=\frac{1}{2} e^{-i \theta}\left(\frac{\partial}{\partial r}-\frac{i}{r} \frac{\partial}{\partial \theta}\right) \\
\frac{\partial}{\partial \alpha} \alpha=\frac{1}{2 r} \frac{\partial}{\partial r} r^{2}-\frac{i}{2} \frac{\partial}{\partial \theta} \\
\frac{\partial^{2}}{\partial \alpha \partial \alpha^{*}}=\frac{1}{4 r^{2}}\left(r \frac{\partial}{\partial r} r \frac{\partial}{\partial r}+\frac{\partial^{2}}{\partial \theta^{2}}\right) \\
\frac{\partial^{2}}{\partial \alpha^{2}}=\frac{1}{4} e^{-2 i \theta}\left(\frac{\partial^{2}}{\partial r^{2}}-\frac{1}{r^{2}} \frac{\partial^{2}}{\partial \theta^{2}}-\frac{2 i}{r} \frac{\partial^{2}}{\partial r \partial \theta}-\frac{1}{r} \frac{\partial}{\partial r}+\frac{2 i}{r^{2}} \frac{\partial}{\partial \theta}\right)
\end{gathered}
$$

The Fokker-Planck equation in phase and amplitude representation is obtained,

$$
\begin{aligned}
\frac{\partial P}{\partial t}= & -\frac{1}{2} \frac{\partial\left(d_{r} P\right)}{\partial r}-\frac{\partial\left(d_{\theta} P\right)}{\partial \theta}+\frac{1}{2 r} \frac{\partial^{2}\left(D_{r \theta} P\right)}{\partial r \partial \theta}+\frac{1}{4 r} \frac{\partial}{\partial r} \frac{1}{r} \frac{\partial\left(D_{r r} P\right)}{\partial r} \\
& +\frac{\partial^{2} D_{\theta \theta}}{\partial \theta^{2}}
\end{aligned}
$$

where we identify drift and diffusion coefficients in the master equation (59) of $P$ representation, i.e.,

$$
\begin{gathered}
d_{r}=r^{2}\left(\rho_{1}+\rho_{1}^{*}+\rho_{2} e^{-2 i \theta}+\rho_{2}^{*} e^{2 i \theta}\right)+\rho_{11} \\
d_{\theta}=-\frac{i}{2}\left(\rho_{1}-\rho_{1}^{*}+\rho_{2} e^{-2 i \theta}-\rho_{2}^{*} e^{2 i \theta}\right)+\frac{1}{2 r^{2}}\left\{\rho_{12} e^{-2 i \theta}-\rho_{12}^{*} e^{2 i \theta}\right\} \\
D_{r \theta}=-i\left\{\rho_{12} e^{-2 i \theta}-\rho_{12}^{*} e^{2 i \theta}\right\} \\
D_{r r}=r^{2}\left\{\rho_{11}+\rho_{12} e^{-2 i \theta}+\rho_{12}^{*} e^{2 i \theta}\right\} \\
D_{\theta \theta}=\frac{1}{4 r^{2}}\left\{\rho_{11}-\rho_{12} e^{-2 i \theta}-\rho_{12}^{*} e^{2 i \theta}\right\}
\end{gathered}
$$

The quantities $\rho_{1}, \rho_{2}, \rho_{11}$, and $\rho_{12}$ are expressed in Eqs. (15)-(18) where we have replaced $\rho_{1}$ by $\rho_{1}-\gamma$ to include the cavity decay terms.

Assuming zero detuning (where $\rho_{1}=0$ ) and the condition $\rho_{2}=\rho_{2}^{*}>0$, the obtained phase drift term from Eq. (59) leads to

$$
\frac{d\langle\theta\rangle}{d t}=\left\langle d_{\theta}\right\rangle \simeq-\rho_{2}\langle\sin 2 \theta\rangle,
$$

which clearly indicates that the phase locking condition $\left\langle d_{\theta}\right\rangle=0$ along with the stability condition $d\left\langle d_{\theta}\right\rangle / d \theta<0$ can be achieved when $\theta=\pi$ [13] in our case. When this condition is satisfied, the diffusion coefficient for the steady state $\left(r^{2}\right.$ $=\bar{n})$ is given by

$$
\begin{aligned}
D_{\theta \theta}= & \frac{1}{4 \bar{n}}\left(-\beta_{11}-\beta_{22}-\beta_{11}^{*}-\beta_{22}^{*}+\alpha_{12}+\alpha_{21}+\alpha_{12}^{*}+\alpha_{21}^{*}\right) \\
= & 4 \Gamma g^{2}\left[8\left|\Omega_{1}\right|^{2}\left|\Omega_{2}\right|^{2}\right. \\
& \times \frac{\operatorname{Re}\left[\Omega_{1} \Omega_{2}\right]+\left(2 \Gamma^{2}+\Omega_{s}\right)\left\{\left|\Omega_{1}\right|^{2}\left|\Omega_{2}\right|^{2}-\Omega_{s} \operatorname{Re}\left[\Omega_{1} \Omega_{2}\right]\right\}}{\left(2 \Gamma^{2}+\Omega_{s}\right) \Omega_{s}-4\left|\Omega_{1}\right|^{2}\left|\Omega_{2}\right|^{2}} .
\end{aligned}
$$

In the last expression we have considered the case where $g_{1}=g_{2}=g$. We plot Eq. (66) for the Rabi frequencies $\Omega_{1}$ and $\Omega_{2}$ in Fig. 4(a) which shows that, apart from the case when any of or both the driving fields equal to zero, there are a set of points where $D_{\theta \theta}=0$ can be achieved. If we consider equal Rabi frequencies than the condition for the diffusion coefficient to be zero is $\Omega=\Gamma / \sqrt{3}$. This condition indicates clearly that the vanishing of the diffusion coefficient is independent of the coupling constants of the cavity fields. Here we present two more graphs of the diffusion coefficient for two important cases of equal Rabi frequency [Fig. 4(b)] and a fixed Rabi frequency [Fig. 4(c)]. In both these cases we draw the graphs showing the relation of the decay constant $\Gamma$ with the Rabi frequency.

\section{CONCLUSION}

We have studied the quantum dynamics of a double$\Lambda$-type single-atom system with particular emphasis on the gain and squeezing of the emitted light. The atomic coherence induced by two driving fields causes the system to behave as a phase sensitive linear amplifier. This is a different behavior from previously studied systems. This result again emphasizes the importance of the way coherence affects the dynamics of the system. In other aspects, we can control the driving fields so that the controlled coherence between the atomic states yields a correlation between the phases of the spontaneously generated photons of the cavity field. With an appropriate choice of parameters this system is shown to satisfy the condition of a two photon correlated-emission laser. Thus we show that the correlated-emission laser can be realized under a certain condition for some values of Rabi frequencies of the driving fields.

\section{ACKNOWLEDGMENTS}

We thank COMSTECH for their support. H.J.K. and H.W.L. are grateful for the financial support of CQP, COMSATS Institute of Information Technology where this work was done. 

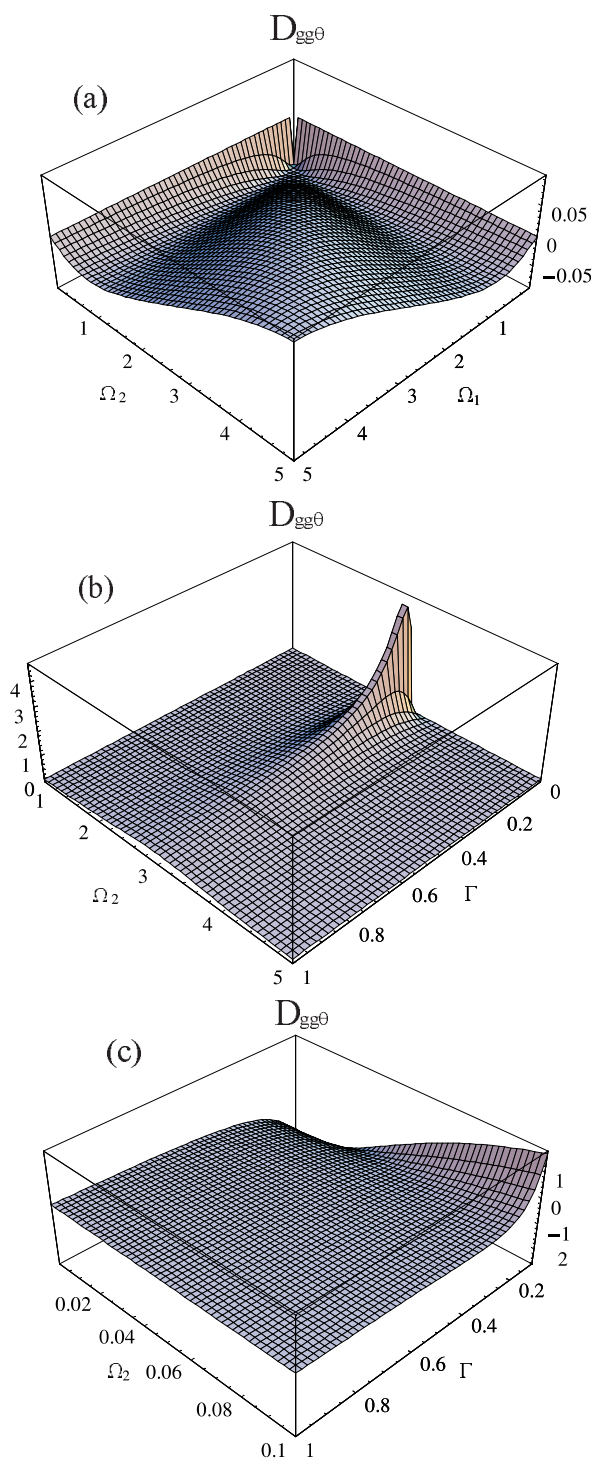

FIG. 4. (Color online) (a) Phase diffusion coefficient $D_{\theta \theta g}$ is plotted for $\Omega_{1}$ and $\Omega_{2}$. We set $\Gamma=1 \mathrm{MHz}, g=1 \mathrm{MHz}$, and $\langle n\rangle=1$. (b) $D_{\theta \theta g}$ vs $\Omega_{2}$ and $\Gamma$, where we take $\Omega_{1}=3$. (c) $D_{\theta \theta g}$ vs equal Rabi frequencies $\Omega$ and $\Gamma$.

\section{APPENDIX: COEFFICIENTS}

Here we give the explicit definitions of the coefficients $a_{i j}$ and $\beta_{i j}$ which enter the master equation (11) for the density operator $\varrho_{F}$ of the two cavity modes:

$$
\begin{aligned}
& \alpha_{11}=2 g_{1}^{2} \Gamma_{2}\left|\Omega_{1}\right|^{2}\left|\Omega_{2}\right|^{2}\left\{4\left(p_{2}^{*}+4 i \Delta_{b}\right)\left|\Omega_{2}\right|^{2}\right. \\
& \left.+p_{1}^{*}\left[4\left|\Omega_{1}\right|^{2}+p_{1}\left(p_{1}+p_{2}^{*}\right)\right]\right\} /\left(p_{3} p_{4}\right), \\
& \beta_{11}=-2 g_{1}^{2} \Gamma_{4}\left|\Omega_{1}\right|^{2}\left|\Omega_{2}\right|^{2}\left\{4 p_{1}\left|\Omega_{2}\right|^{2}+p_{2}^{*}\left[4\left|\Omega_{1}\right|^{2}\right.\right. \\
& \left.\left.+p_{1}\left(p_{1}+p_{2}^{*}\right)\right]\right\} /\left(p_{3} p_{4}\right) \text {, } \\
& \alpha_{12}=-2 g_{1} g_{2} \Gamma_{2} \Omega_{1} \Omega_{2}\left|\Omega_{1}\right|^{2}\left\{4 p_{1}\left|\Omega_{1}\right|^{2}\right. \\
& \left.+p_{1}^{2}\left(p_{1}+p_{2}^{*}\right)-4\left|\Omega_{2}\right|^{2}\left(2 p_{1}+p_{2}^{*}\right)\right\} /\left(p_{3} p_{4}\right), \\
& \beta_{12}=-2 g_{1} g_{2} \Gamma_{4} \Omega_{1} \Omega_{2}\left|\Omega_{2}\right|^{2}\left[\left(p_{1}+p_{2}^{*}\right)\left|p_{2}\right|^{2}+4\left|\Omega_{2}\right|^{2} p_{2}\right. \\
& \left.+4\left|\Omega_{1}\right|^{2}\left(p_{1}-4 i \Delta_{a}\right)\right] /\left(p_{3} p_{4}\right), \\
& \alpha_{22}=2 g_{2}^{2} \Gamma_{4}\left|\Omega_{1}\right|^{2}\left|\Omega_{2}\right|^{2}\left\{4\left(p_{1}^{*}+4 i \Delta_{a}\right)\left|\Omega_{1}\right|^{2}\right. \\
& \left.+p_{2}^{*}\left[4\left|\Omega_{2}\right|^{2}+p_{2}\left(p_{2}+p_{1}^{*}\right)\right]\right\} /\left(p_{3} p_{5}\right), \\
& \beta_{22}=-2 g_{2}^{2} \Gamma_{2}\left|\Omega_{1}\right|^{2}\left|\Omega_{2}\right|^{2}\left\{4 p_{2}\left|\Omega_{1}\right|^{2}+p_{1}^{*}\left[4\left|\Omega_{2}\right|^{2}\right.\right. \\
& \left.\left.+p_{2}\left(p_{2}+p_{1}^{*}\right)\right]\right\} /\left(p_{3} p_{5}\right) \\
& \alpha_{21}=-2 g_{1} g_{2} \Gamma_{4} \Omega_{1} \Omega_{2}\left|\Omega_{2}\right|^{2}\left\{4 p_{2}\left|\Omega_{2}\right|^{2}+p_{2}^{2}\left(p_{2}+p_{1}^{*}\right)\right. \\
& \left.-4\left|\Omega_{1}\right|^{2}\left(2 p_{2}+p_{1}^{*}\right)\right\} /\left(p_{3} p_{5}\right), \\
& \beta_{21}=-2 g_{1} g_{2} \Gamma_{2} \Omega_{1} \Omega_{2}\left|\Omega_{2}\right|^{2}\left[\left(p_{2}+p_{1}^{*}\right)\left|p_{1}\right|^{2}+4\left|\Omega_{1}\right|^{2} p_{1}\right. \\
& \left.+4\left|\Omega_{2}\right|^{2}\left(p_{2}-4 i \Delta_{b}\right)\right] /\left(p_{3} p_{5}\right),
\end{aligned}
$$

The parameters $p_{1}, p_{2}, p_{3}, p_{4}$, and $p_{5}$ in Eqs. (A1)-(A8) are defined as

$$
\begin{gathered}
p_{1}=\Gamma_{3}+\Gamma_{4}+2 i \Delta_{b}, \\
p_{2}=\Gamma_{1}+\Gamma_{2}+2 i \Delta_{a}, \\
p_{3}=\Gamma_{2}\left|p_{1}\right|^{2}\left|\Omega_{1}\right|^{2}+\Gamma_{4}\left|p_{2}\right|^{2}\left|\Omega_{2}\right|^{2}+8\left|\Omega_{1}\right|^{2}\left|\Omega_{2}\right|^{2}\left(\Gamma_{2}+\Gamma_{4}\right),
\end{gathered}
$$

$p_{4}=4\left(\left|\Omega_{1}\right|^{2}-\left|\Omega_{2}\right|^{2}\right)^{2}+p_{1}\left(p_{1}+p_{2}^{*}\right)\left|\Omega_{1}\right|^{2}+p_{2}^{*}\left(p_{1}+p_{2}^{*}\right)\left|\Omega_{2}\right|^{2}$,

$p_{5}=4\left(\left|\Omega_{1}\right|^{2}-\left|\Omega_{2}\right|^{2}\right)^{2}+p_{1}^{*}\left(p_{2}+p_{1}^{*}\right)\left|\Omega_{1}\right|^{2}+p_{2}\left(p_{2}+p_{1}^{*}\right)\left|\Omega_{2}\right|^{2}$.
[1] Y. Mu and C. M. Savage, Phys. Rev. A 46, 5944 (1992).

[2] C. Ginzel, H. J. Briegel, U. Martini, B. G. Englert, and A. Schenzle, Phys. Rev. A 48, 732 (1993).

[3] P. Horak, K. M. Gheri, and H. Ritsch, Phys. Rev. A 51, 3257 (1995).

[4] M. Loffler, G. M. Meyer, and H. Walther, Phys. Rev. A 55, 3923 (1997).

[5] B. Jones, S. Ghose, J. P. Clemens, P. R. Rice, and L. M.
Pedrotti, Phys. Rev. A 60, 3267 (1999).

[6] K. An, J. J. Childs, R. R. Dasari, and M. S. Feld, Phys. Rev. Lett. 73, 3375 (1994).

[7] G. M. Meyer, M. Loffler, and H. Walther, Phys. Rev. A 56, R1099 (1997).

[8] G. M. Meyer and H. J. Briegel, Phys. Rev. A 58, 3210 (1998).

[9] J. McKeever, A. Boca, A. D. Boozer, J. R. Buck, and H. J. Kimble, Nature (London) 425, 268 (2003). 
[10] A. D. Boozer, A. Boca, J. R. Buck, J. McKeever, and H. J. Kimble, Phys. Rev. A 70, 023814 (2004).

[11] T. Pellizzari and H. Ritsch, Phys. Rev. Lett. 72, 3973 (1994).

[12] S. Ya. Kilin and T. B. Karlovich, JETP 95, 805 (2002).

[13] N. A. Ansari, J. Gea-Banacloche, and M. S. Zubairy, Phys. Rev. A 41, 5179 (1990).

[14] M. Kiffner, M. S. Zubairy, J. Evers, and C. H. Keitel, Phys. Rev. A 75, 033816 (2007).

[15] A. L. Schawlow and C. H. Townes, Phys. Rev. 112, 1940 (1958).

[16] M. O. Scully and M. S. Zubairy, Quantum Optics (Cambridge University Press, Cambridge, England, 1997).

[17] M. O. Scully, Phys. Rev. Lett. 55, 2802 (1985); M. O. Scully and M. S. Zubairy, Phys. Rev. A 35, 752 (1987); Opt. Commun. 66, 303 (1988); M. O. Scully, K. Wodkiewicz, M. S. Zubairy, J. Bergou, N. Lu, and J. Meyer ter Vehn, Phys. Rev. Lett. 60, 1832 (1988).

[18] J. Bergou, J. Zhang, and C. Su, Phys. Rev. A 52, 3183 (1995).

[19] R. J. Glauber, Phys. Rev. 130, 2529 (1963); 131, 2766 (1963);

[20] K. Zaheer and M. S. Zubairy, Phys. Rev. A 38, 5227 (1988); Nadeem A. Ansari and M. S. Zubairy, ibid. 40, 5690 (1989); Ning Lu, ibid. 45, 8154 (1992).

[21] H. Xiong, M. O. Scully, and M. S. Zubairy, Phys. Rev. Lett. 94, 023601 (2005).

[22] H.-T. Tan, S.-Y. Zhu, and M. S. Zubairy, Phys. Rev. A 72, 022305 (2005). 